\title{
Differential Sandwich Theorems for a Certain Class of Analytic Functions Defined by Differential Operator
}

\author{
Abbas Kareem Wanas ${ }^{1}$ and Hala Abbas Mehai ${ }^{2}$ \\ ${ }^{1}$ Department of Mathematics, College of Science, University of Al-Qadisiyah, Iraq; \\ e-mail: abbas.kareem.w@qu.edu.iq \\ ${ }^{2}$ Department of Mathematics, College of Computer Science and Information Technology, \\ University of Al-Qadisiyah, Iraq; e-mail: halahaider2015@gmail.com
}

\begin{abstract}
In this paper, we establish some applications of first order differential subordination and superordination results involving Hadamard product for a certain class of analytic functions with differential operator defined in the open unit disk. These results are applied to obtain sandwich results.
\end{abstract}

\section{Introduction and Preliminaries}

Let $\mathcal{H}$ indicate the family of analytic functions in the open unit disk $U=\{z \in \mathbb{C}:|z|<1\}$ and let $\mathcal{H}[a, p]$ be the subclass of $\mathcal{H}$ consisting of functions of the form:

$$
f(z)=a+a_{p} z^{p}+a_{p+1} z^{p+1}+\cdots, \quad(a \in \mathbb{C}, p \in \mathbb{N}=\{1,2, \ldots\}) .
$$

Also, let $\mathcal{A}$ denote the subclass of $\mathcal{H}$ consisting of functions of the form:

$$
f(z)=z+\sum_{n=2}^{\infty} a_{n} z^{n},
$$

Received: February 22, 2019; Accepted: March 24, 2019

2010 Mathematics Subject Classification: 30C45.

Keywords and phrases: analytic functions, differential subordination, differential superordination, Hadamard product, differential operator.

Copyright (C) 2019 Abbas Kareem Wanas and Hala Abbas Mehai. This is an open access article distributed under the Creative Commons Attribution License, which permits unrestricted use, distribution, and reproduction in any medium, provided the original work is properly cited. 
Let $f, g \in \mathcal{H}$. The function $f$ is said to be subordinate to $g$, or $g$ is said to be superordinate to $f$, if there exists a Schwarz function $w$ analytic in $U$ with $w(0)=0$ and $|w(z)|<1(z \in U)$ such that $f(z)=g(w(z))$. This subordination is denoted by $f \prec g$ or $f(z) \prec g(z)(z \in U)$. It is well known that, if the function $g$ is univalent in $U$, we have the following equivalence (see [12]):

$$
f \prec g(z \in U) \Leftrightarrow f(0)=g(0), \quad f(U) \subset g(U) .
$$

Let $k, h \in \mathcal{H}$ and $\psi(r, s, t ; z): \mathbb{C}^{3} \times U \rightarrow \mathbb{C}$. If $k$ and $\psi\left(k(z), z k^{\prime}(z), z^{2} k^{\prime \prime}(z) ; z\right)$ are univalent functions in $U$ and if $k$ satisfies the second-order differential superordination:

$$
h(z) \prec \psi\left(k(z), z k^{\prime}(z), z^{2} k^{\prime \prime}(z) ; z\right),
$$

then $k$ is called a solution of the differential superordination (1.2). (If $f$ is subordinate to $g$, then $g$ is superordinate to $f$ ). An analytic function $q$ is called a subordinant of (1.2), if $q \prec k$ for all $k$ satisfying (1.2). A univalent subordinant $\tilde{q}$ that satisfies $q \prec \tilde{q}$ for all the subordinants $q$ of (1.2) is called the best subordinant.

For the functions $f \in \mathcal{A}$ given by (1.1) and $g \in \mathcal{A}$ defined by

$$
g(z)=z+\sum_{n=2}^{\infty} b_{n} z^{n}
$$

we define the Hadamard product (or convolution) $f * g$ of the functions $f$ and $g$ (as usual) by

$$
(f * g)(z)=z+\sum_{k=2}^{\infty} a_{n} b_{n} z^{n}=(g * f)(z) .
$$

For $m \in \mathbb{N}_{0}=\mathbb{N} \cup\{0\}, \quad \rho, \lambda \geq 0, \quad \mu, v, \delta>0$ and $\rho \neq v$, we consider the differential operator $A_{\mu, v, \lambda}^{m}(\rho, \delta): \mathcal{A} \rightarrow \mathcal{A}$, introduced by Amourah and Darus [2], where

$$
A_{\mu, v, \lambda}^{m}(\rho, \delta) f(z)=z+\sum_{n=2}^{\infty}\left[1+\frac{(n-1)[(v-\rho) \delta+n \lambda]}{\mu+v}\right]^{m} a_{n} z^{n}
$$


It is readily verified from (1.3) that

$$
\begin{aligned}
z\left(A_{\mu, v, \lambda}^{m}(\rho, \delta) f(z)\right)^{\prime}= & \frac{\mu+v}{(v-\rho) \delta+n \lambda} A_{\mu, v, \lambda}^{m+1}(\rho, \delta) f(z) \\
& -\left(1-\frac{\mu+v}{(v-\rho) \delta+n \lambda}\right) A_{\mu, v, \lambda}^{m}(\rho, \delta) f(z) .
\end{aligned}
$$

Would like to point out here that some of the special cases of the operator defined by (1.3) can be found in $[1,4,11,13]$.

Recently several authors, Goyal et al. [5], Murugusundaramoorthy and Magesh [9, 10], Magesh et al. [7], Ibrahim and Darus [6], Wanas [14, 15], Wanas and Joudah [16] and Wanas and Majeed [17] have obtained sandwich results for certain classes of analytic functions.

The main object of the present investigation is to find sufficient condition for certain normalized analytic functions $f$ in $U$ such that $(f * \Psi)(z) \neq 0$ and $f$ to satisfy

$$
q_{1}(z) \prec\left(\frac{A_{\mu, v, \lambda}^{m+1}(\rho, \delta)(f * \Phi)(z)}{A_{\mu, v, \lambda}^{m}(\rho, \delta)(f * \Psi)(z)}\right)^{\gamma} \prec q_{2}(z),
$$

where $q_{1}$ and $q_{2}$ are given univalent functions in $U$ with $q_{1}(0)=q_{2}(0)=1$ and $\Phi(z)=z+\sum_{n=2}^{\infty} r_{n} z^{n}, \quad \Psi(z)=z+\sum_{n=2}^{\infty} e_{n} z^{n}$ are analytic functions in $U$ with $r_{n} \geq 0, e_{n} \geq 0$.

To establish our main results, we need the following definition and lemmas.

Definition 1.1 [8]. Denote by $Q$ the set of all functions $f$ that are analytic and injective on $\bar{U} \backslash E(f)$, where

$$
E(f)=\left\{\zeta \in \partial U: \lim _{z \rightarrow \zeta} f(z)=\infty\right\}
$$

and are such that $f^{\prime}(\zeta) \neq 0$ for $\zeta \in \partial U \backslash E(f)$.

Lemma 1.1 [8]. Let $q$ be univalent in the unit disk $U$ and let $\theta$ and $\phi$ be analytic in a domain D containing $q(U)$ with $\phi(w) \neq 0$ when $w \in q(U)$. Set $Q(z)=z q^{\prime}(z) \phi(q(z))$ and $h(z)=\theta(q(z))+Q(z)$. Suppose that 
(1) $Q(z)$ is starlike univalent in $U$,

(2) $\operatorname{Re}\left\{\frac{z h^{\prime}(z)}{Q(z)}\right\}>0$ for $z \in U$.

If $k$ is analytic in $U$, with $k(0)=q(0), k(U) \subset D$ and

$$
\theta(k(z))+z k^{\prime}(z) \phi(k(z)) \prec \theta(q(z))+z q^{\prime}(z) \phi(q(z)),
$$

then $k \prec q$ and $q$ is the best dominant of (1.5).

Lemma 1.2 [3]. Let $q$ be convex univalent in the unit disk $U$ and let $\theta$ and $\phi$ be analytic in a domain $D$ containing $q(U)$. Suppose that

(1) $\operatorname{Re}\left\{\frac{\theta^{\prime}(q(z))}{\phi(q(z))}\right\}>0$ for $z \in U$,

(2) $Q(z)=z q^{\prime}(z) \phi(q(z))$ is starlike univalent in $U$.

If $k \in \mathcal{H}[q(0), 1] \cap Q$, with $k(U) \subset D, \theta(k(z))+z k^{\prime}(z) \phi(k(z))$ is univalent in $U$ and

$$
\theta(q(z))+z q^{\prime}(z) \phi(q(z)) \prec \theta(k(z))+z k^{\prime}(z) \phi(k(z)),
$$

then $q \prec k$ and $q$ is the best subordinant of (1.6).

\section{Main Results}

Theorem 2.1. Let $\Phi, \Psi \in \mathcal{A}, \quad \alpha, \beta, \tau \in \mathbb{C}, \eta, \gamma \in \mathbb{C} \backslash\{0\}$ and let $q$ be convex univalent in $U$ with $q(0)=1$ and assume that $q$ satisfies:

$$
\operatorname{Re}\left\{1+\frac{\alpha \tau}{\eta}+\frac{\beta(\tau+1)}{\eta} q(z)+(\tau-1) \frac{z q^{\prime}(z)}{q(z)}+\frac{z q^{\prime \prime}(z)}{q^{\prime}(z)}\right\}>0 .
$$

Suppose that $z(q(z))^{\tau-1} q^{\prime}(z)$ is starlike univalent in $U$. If $f \in \mathcal{A}_{p}$ satisfies the differential subordination:

$$
\begin{aligned}
& \varphi_{1}(f, \Phi, \Psi, \alpha, \beta, \tau, \eta, \gamma, \rho, \delta, \mu, v, \lambda, m ; z) \\
\prec & (\alpha+\beta q(z))(q(z))^{\tau}+\eta z(q(z))^{\tau-1} q^{\prime}(z),
\end{aligned}
$$


where

$$
\begin{aligned}
& \varphi_{1}(f, \Phi, \Psi, \alpha, \beta, \tau, \eta, \gamma, \rho, \delta, \mu, v, \lambda, m ; z) \\
= & \alpha\left(\frac{A_{\mu, v, \lambda}^{m+1}(\rho, \delta)(f * \Phi)(z)}{A_{\mu, v, \lambda}^{m}(\rho, \delta)(f * \Psi)(z)}\right)^{\gamma \tau}+\beta\left(\frac{A_{\mu, v, \lambda}^{m+1}(\rho, \delta)(f * \Phi)(z)}{A_{\mu, v, \lambda}^{m}(\rho, \delta)(f * \Psi)(z)}\right)^{\gamma(\tau+1)} \\
+ & \frac{\eta \eta \mu+v)}{(v-\rho) \delta+n \lambda}\left(\frac{A_{\mu, v, \lambda}^{m+1}(\rho, \delta)(f * \Phi)(z)}{A_{\mu, v, \lambda}^{m}(\rho, \delta)(f * \Psi)(z)}\right)^{\gamma \tau}\left(\frac{A_{\mu, v, \lambda}^{m+2}(\rho, \delta)(f * \Phi)(z)}{A_{\mu, v, \lambda}^{m+1}(\rho, \delta)(f * \Phi)(z)}\right. \\
- & \left.\frac{A_{\mu, v, \lambda}^{m+1}(\rho, \delta)(f * \Psi)(z)}{A_{\mu, v, \lambda}^{m}(\rho, \delta)(f * \Psi)(z)}\right)
\end{aligned}
$$

then

$$
\left(\frac{A_{\mu, v, \lambda}^{m+1}(\rho, \delta)(f * \Phi)(z)}{A_{\mu, \nu, \lambda}^{m}(\rho, \delta)(f * \Psi)(z)}\right)^{\gamma} \prec q(z)
$$

and $q$ is the best dominant of (2.2).

Proof. Let the function $k$ be defined by

$$
k(z)=\left(\frac{A_{\mu, v, \lambda}^{m+1}(\rho, \delta)(f * \Phi)(z)}{A_{\mu, \nu, \lambda}^{m}(\rho, \delta)(f * \Psi)(z)}\right)^{\gamma}, \quad(z \in U) .
$$

Then the function $k$ is analytic in $U$ and $k(0)=1$.

A simple computation using (2.4) gives

$$
\frac{z k^{\prime}(z)}{k(z)}=\gamma\left(\frac{z\left(A_{\mu, v, \lambda}^{m+1}(\rho, \delta)(f * \Phi)(z)\right)^{\prime}}{A_{\mu, v, \lambda}^{m+1}(\rho, \delta)(f * \Phi)(z)}-\frac{z\left(A_{\mu, v, \lambda}^{m}(\rho, \delta)(f * \Psi)(z)\right)^{\prime}}{A_{\mu, \nu, \lambda}^{m}(\rho, \delta)(f * \Psi)(z)}\right) .
$$

In view of (1.4), we obtain

$$
\frac{z k^{\prime}(z)}{k(z)}=\frac{\gamma(\mu+v)}{(v-\rho) \delta+n \lambda}\left(\frac{A_{\mu, v, \lambda}^{m+2}(\rho, \delta)(f * \Phi)(z)}{A_{\mu, v, \lambda}^{m+1}(\rho, \delta)(f * \Phi)(z)}-\frac{A_{\mu, v, \lambda}^{m+1}(\rho, \delta)(f * \Psi)(z)}{A_{\mu, v, \lambda}^{m}(\rho, \delta)(f * \Psi)(z)}\right)
$$


Also, we find that

$$
\begin{aligned}
& (\alpha+\beta k(z))(k(z))^{\tau}+\eta z(k(z))^{\tau-1} k^{\prime}(z) \\
= & \varphi_{1}(f, \Phi, \Psi, \alpha, \beta, \tau, \eta, \gamma, \rho, \delta, \mu, v, \lambda, m ; z),
\end{aligned}
$$

where $\varphi_{1}(f, \Phi, \Psi, \alpha, \beta, \tau, \eta, \gamma, \rho, \delta, \mu, v, \lambda, m ; z)$ is given by (2.3).

By using (2.5) in (2.2), we have

$$
(\alpha+\beta k(z))(k(z))^{\tau}+\eta z(k(z))^{\tau-1} k^{\prime}(z) \prec(\alpha+\beta q(z))(q(z))^{\tau}+\eta z(q(z))^{\tau-1} q^{\prime}(z) .
$$

By setting

$$
\theta(w)=(\alpha+\beta w) w^{\tau} \text { and } \phi(w)=\eta w^{\tau-1}, \quad w \neq 0,
$$

it can be easily observed that $\theta(w)$ is analytic in $\mathbb{C}, \phi(w)$ is analytic in $\mathbb{C} \backslash\{0\}$ and that $\phi(w) \neq 0, w \in \mathbb{C} \backslash\{0\}$. Also, we get

$$
Q(z)=z q^{\prime}(z) \phi(q(z))=\eta z(q(z))^{\tau-1} q^{\prime}(z)
$$

and

$$
h(z)=\theta(q(z))+Q(z)=(\alpha+\beta q(z))(q(z))^{\tau}+\eta z(q(z))^{\tau-1} q^{\prime}(z) .
$$

In light of the hypothesis of Theorem 2.1, we see that $Q(z)$ is starlike univalent in $U$ and

$$
\operatorname{Re}\left\{\frac{z h^{\prime}(z)}{Q(z)}\right\}=\operatorname{Re}\left\{1+\frac{\alpha \tau}{\eta}+\frac{\beta(\tau+1)}{\eta} q(z)+(\tau-1) \frac{z q^{\prime}(z)}{q(z)}+\frac{z q^{\prime \prime}(z)}{q^{\prime}(z)}\right\}>0 .
$$

Hence the result now follows by an application of Lemma 1.1.

By taking $q(z)=\frac{1+A z}{1+B z}(-1 \leq B<A \leq 1)$ in Theorem 2.1, we obtain the following corollary:

Corollary 2.1. Let $\Phi, \Psi \in \mathcal{A}, \alpha, \beta, \tau \in \mathbb{C}, \eta, \gamma \in \mathbb{C} \backslash\{0\},-1 \leq B<A \leq 1$ and assume that

$$
\operatorname{Re}\left\{\frac{\alpha \tau}{\eta}+\frac{\beta(\tau+1)(1+A z)}{\eta(1+B z)}+\frac{1+\tau(A-B) z-A B z^{2}}{(1+A z)(1+B z)}\right\}>0
$$


If $f \in \mathcal{A}$ satisfies the differential subordination:

$$
\begin{aligned}
& \varphi_{1}(f, \Phi, \Psi, \alpha, \beta, \tau, \eta, \gamma, \rho, \delta, \mu, \nu, \lambda, m ; z) \\
\prec & \left(\alpha+\beta\left(\frac{1+A z}{1+B z}\right)\right)\left(\frac{1+A z}{1+B z}\right)^{\tau}+\frac{\eta(A-B)(1+A z)^{\tau-1} z}{(1+B z)^{\tau+1}},
\end{aligned}
$$

where $\varphi_{1}(f, \Phi, \Psi, \alpha, \beta, \tau, \eta, \gamma, \rho, \delta, \mu, v, \lambda, m ; z)$ is given by (2.3), then

$$
\left(\frac{A_{\mu, v, \lambda}^{m+1}(\rho, \delta)(f * \Phi)(z)}{A_{\mu, v, \lambda}^{m}(\rho, \delta)(f * \Psi)(z)}\right)^{\gamma} \prec \frac{1+A z}{1+B z}
$$

and $q(z)=\frac{1+A z}{1+B z}$ is the best dominant of (2.6).

By fixing $\Phi(z)=\Psi(z)=\frac{z}{1-z}$ in Theorem 2.1, we obtain the following corollary:

Corollary 2.2. Let $\alpha, \beta, \tau \in \mathbb{C}, \eta, \gamma \in \mathbb{C} \backslash\{0\}$ and let $q$ be convex univalent in $U$ with $q(0)=1$ and assume that (2.1) holds true. Suppose that $z(q(z))^{\tau-1} q^{\prime}(z)$ is starlike univalent in $U$. If $f \in \mathcal{A}$ satisfies the differential subordination:

$$
\varphi_{2}(f, \alpha, \beta, \tau, \eta, \gamma, \rho, \delta, \mu, \nu, \lambda, m ; z) \prec(\alpha+\beta q(z))(q(z))^{\tau}+\eta z(q(z))^{\tau-1} q^{\prime}(z),(2.7)
$$

where

$$
\begin{aligned}
& \varphi_{2}(f, \alpha, \beta, \tau, \eta, \gamma, \rho, \delta, \mu, v, \lambda, m ; z) \\
= & \alpha\left(\frac{A_{\mu, v, \lambda}^{m+1}(\rho, \delta) f(z)}{A_{\mu, v, \lambda}^{m}(\rho, \delta) f(z)}\right)^{\gamma \tau}+\beta\left(\frac{A_{\mu, v, \lambda}^{m+1}(\rho, \delta) f(z)}{A_{\mu, v, \lambda}^{m}(\rho, \delta) f(z)}\right)^{\gamma(\tau+1)} \\
+ & \frac{\eta(\mu+v)}{(v-\rho) \delta+n \lambda}\left(\frac{A_{\mu, v, \lambda}^{m+1}(\rho, \delta) f(z)}{A_{\mu, v, \lambda}^{m}(\rho, \delta) f(z)}\right)^{\gamma \tau}\left(\frac{A_{\mu, v, \lambda}^{m+2}(\rho, \delta) f(z)}{A_{\mu, v, \lambda}^{m+1}(\rho, \delta) f(z)}-\frac{A_{\mu, v, \lambda}^{m+1}(\rho, \delta) f(z)}{A_{\mu, v, \lambda}^{m}(\rho, \delta) f(z)}\right),
\end{aligned}
$$

then

$$
\left(\frac{A_{\mu, v, \lambda}^{m+1}(\rho, \delta) f(z)}{A_{\mu, v, \lambda}^{m}(\rho, \delta) f(z)}\right)^{\gamma} \prec q(z)
$$

and $q$ is the best dominant of (2.7). 
Theorem 2.2. Let $\Phi, \Psi \in \mathcal{A}, \quad \alpha, \beta, \tau \in \mathbb{C}, \eta, \gamma \in \mathbb{C} \backslash\{0\}$ and let $q$ be convex univalent in $U$ with $q(0)=1$ and assume that $q$ satisfies:

$$
\operatorname{Re}\left\{\frac{\alpha \tau}{\eta} q^{\prime}(z)+\frac{\beta(\tau+1)}{\eta} q(z) q^{\prime}(z)\right\}>0 .
$$

Suppose that $z(q(z))^{\tau-1} q^{\prime}(z)$ is starlike univalent in $U$. Let $f \in \mathcal{A}$ satisfy

$$
\left(\frac{A_{\mu, \nu, \lambda}^{m+1}(\rho, \delta)(f * \Phi)(z)}{A_{\mu, \nu, \lambda}^{m}(\rho, \delta)(f * \Psi)(z)}\right)^{\gamma} \in \mathcal{H}[q(0), 1] \cap Q
$$

and $\varphi_{1}(f, \Phi, \Psi, \alpha, \beta, \tau, \eta, \gamma, \rho, \delta, \mu, v, \lambda, m ; z)$ as defined by (2.3) be univalent in $U$. If

$$
\begin{gathered}
(\alpha+\beta q(z))(q(z))^{\tau}+\eta_{z}(q(z))^{\tau-1} q^{\prime}(z) \\
\prec \varphi_{1}(f, \Phi, \Psi, \alpha, \beta, \tau, \eta, \gamma, \rho, \delta, \mu, \nu, \lambda, m ; z),
\end{gathered}
$$

then

$$
q(z) \prec\left(\frac{A_{\mu, \nu, \lambda}^{m+1}(\rho, \delta)(f * \Phi)(z)}{A_{\mu, \nu, \lambda}^{m}(\rho, \delta)(f * \Psi)(z)}\right)^{\gamma}
$$

and $q$ is the best subordinant of (2.10).

Proof. Let the function $k$ be defined by (2.4).

In view of (1.4), the superordination (2.10) becomes

$$
(\alpha+\beta q(z))(q(z))^{\tau}+\eta z(q(z))^{\tau-1} q^{\prime}(z) \prec(\alpha+\beta k(z))(k(z))^{\tau}+\eta z(k(z))^{\tau-1} k^{\prime}(z) .
$$

By setting $\theta(w)=(\alpha+\beta w) w^{\tau}$ and $\phi(w)=\eta w^{\tau-1}, w \neq 0$, it is easily observed that $\theta(w)$ is analytic in $\mathbb{C}, \phi(w)$ is analytic in $\mathbb{C} \backslash\{0\}$ and that $\phi(w) \neq 0, w \in \mathbb{C} \backslash\{0\}$. Also, we get

$$
Q(z)=z q^{\prime}(z) \phi(q(z))=\eta z(q(z))^{\tau-1} q^{\prime}(z) .
$$

It is clear that $Q(z)$ is starlike univalent in $U$ and 


$$
\operatorname{Re}\left\{\frac{\theta^{\prime}(q(z))}{\phi(q(z))}\right\}=\operatorname{Re}\left\{\frac{\alpha \tau}{\eta} q^{\prime}(z)+\frac{\beta(\tau+1)}{\eta} q(z) q^{\prime}(z)\right\}>0 .
$$

Now Theorem 2.2 follows by applying Lemma 1.2.

By fixing $\Phi(z)=\Psi(z)=\frac{z}{1-z}$ in Theorem 2.2, we obtain the following corollary:

Corollary 2.3. Let $\alpha, \beta, \tau \in \mathbb{C}, \eta, \gamma \in \mathbb{C} \backslash\{0\}$ and let $q$ be convex univalent in $U$ with $q(0)=1$ and assume that (2.9) holds true. Suppose that $z(q(z))^{\tau-1} q^{\prime}(z)$ is starlike univalent in $U$. Let $f \in \mathcal{A}$ satisfy

$$
\left(\frac{A_{\mu, \nu, \lambda}^{m+1}(\rho, \delta) f(z)}{A_{\mu, \nu, \lambda}^{m}(\rho, \delta) f(z)}\right)^{\gamma} \in \mathcal{H}[q(0), 1] \cap Q
$$

and $\varphi_{2}(f, \alpha, \beta, \tau, \eta, \gamma, \rho, \delta, \mu, \nu, \lambda, m ; z)$ as defined by (2.8) be univalent in $U$. If $(\alpha+\beta q(z))(q(z))^{\tau}+\eta z(q(z))^{\tau-1} q^{\prime}(z) \prec \varphi_{2}(f, \alpha, \beta, \tau, \eta, \gamma, \rho, \delta, \mu, v, \lambda, m ; z),(2.11)$ then

$$
q(z) \prec\left(\frac{A_{\mu, \nu, \lambda}^{m+1}(\rho, \delta) f(z)}{A_{\mu, \nu, \lambda}^{m}(\rho, \delta) f(z)}\right)^{\gamma}
$$

and $q$ is the best subordinant of (2.11).

Concluding the results of differential subordination and superordination, we arrive at the following "sandwich results".

Theorem 2.3. Let $q_{1}$ and $q_{2}$ be convex univalent in $U$ with $q_{1}(0)=q_{2}(0)=1$, $\alpha, \beta, \tau \in \mathbb{C}, \eta, \gamma \in \mathbb{C} \backslash\{0\}$. Suppose $q_{2}$ satisfies (2.1) and $q_{1}$ satisfies (2.9) such that $z(q(z))^{\tau-1} q^{\prime}(z)$ is starlike univalent in $U$. For $f, \Phi, \Psi \in \mathcal{A}$, let

$$
\left(\frac{A_{\mu, v, \lambda}^{m+1}(\rho, \delta)(f * \Phi)(z)}{A_{\mu, \nu, \lambda}^{m}(\rho, \delta)(f * \Psi)(z)}\right)^{\gamma} \in \mathcal{H}[1,1] \cap Q
$$

and $\varphi_{1}(f, \Phi, \Psi, \alpha, \beta, \tau, \eta, \gamma, \rho, \delta, \mu, v, \lambda, m ; z)$ as defined by (2.3) be univalent in $U$. 
If

$$
\begin{aligned}
& \left(\alpha+\beta q_{1}(z)\right)\left(q_{1}(z)\right)^{\tau}+\eta z\left(q_{1}(z)\right)^{\tau-1} q_{1}^{\prime}(z) \\
\prec & \varphi_{1}(f, \Phi, \Psi, \alpha, \beta, \tau, \eta, \gamma, \rho, \delta, \mu, \nu, \lambda, m ; z) \\
\prec & \left(\alpha+\beta q_{2}(z)\right)\left(q_{2}(z)\right)^{\tau}+\eta z\left(q_{2}(z)\right)^{\tau-1} q_{2}^{\prime}(z),
\end{aligned}
$$

then

$$
q_{1}(z) \prec\left(\frac{A_{\mu, v, \lambda}^{m+1}(\rho, \delta)(f * \Phi)(z)}{A_{\mu, \nu, \lambda}^{m}(\rho, \delta)(f * \Psi)(z)}\right)^{\gamma} \prec q_{2}(z)
$$

and $q_{1}, q_{2}$ are respectively the best subordinant and the best dominant.

By making use of Corollaries 2.2 and 2.3, we obtain the following corollary:

Corollary 2.4. Let $q_{1}$ and $q_{2}$ be convex univalent in $U$ with $q_{1}(0)=q_{2}(0)=1$, $\alpha, \beta, \tau \in \mathbb{C}, \eta, \gamma \in \mathbb{C} \backslash\{0\}$. Suppose $q_{2}$ satisfies (2.1) and $q_{1}$ satisfies (2.9) such that $z(q(z))^{\tau-1} q^{\prime}(z)$ is starlike univalent in $U$. For $f \in \mathcal{A}$, let

$$
\left(\frac{A_{\mu, \nu, \lambda}^{m+1}(\rho, \delta) f(z)}{A_{\mu, \nu, \lambda}^{m}(\rho, \delta) f(z)}\right)^{\gamma} \in \mathcal{H}[1,1] \cap Q
$$

and $\varphi_{2}(f, \alpha, \beta, \tau, \eta, \gamma, \rho, \delta, \mu, v, \lambda, m ; z)$ as defined by (2.8) be univalent in $U$. If

$$
\begin{aligned}
& \left(\alpha+\beta q_{1}(z)\right)\left(q_{1}(z)\right)^{\tau}+\eta z\left(q_{1}(z)\right)^{\tau-1} q_{1}^{\prime}(z) \\
\prec & \varphi_{2}(f, \alpha, \beta, \tau, \eta, \gamma, \rho, \delta, \mu, v, \lambda, m ; z) \\
\prec & \left(\alpha+\beta q_{2}(z)\right)\left(q_{2}(z)\right)^{\tau}+\eta z\left(q_{2}(z)\right)^{\tau-1} q_{2}^{\prime}(z),
\end{aligned}
$$

then

$$
q_{1}(z) \prec\left(\frac{A_{\mu, v, \lambda}^{m+1}(\rho, \delta) f(z)}{A_{\mu, v, \lambda}^{m}(\rho, \delta) f(z)}\right)^{\gamma} \prec q_{2}(z)
$$

and $q_{1}, q_{2}$ are respectively the best subordinant and the best dominant. 


\section{References}

[1] F. M. Al-Oboudi, On univalent functions defined by a generalized Sălăgean operator, Int. J. Math. Math. Sci. 27 (2004), 1429-1436.

[2] A. Amourah and M. Darus, Some properties of a new class of univalent functions involving a new generalized differential operator with negative coefficients, Indian J. Sci. Tech. 9(36) (2016), 1-7.

[3] T. Bulboacă, Classes of first-order differential superordinations, Demonstratio Math. 35(2) (2002), 287-292.

[4] M. Darus and R. W. Ibrahim, On subclasses for generalized operators of complex order, Far East J. Math. Sci. (FJMS) 33(3) (2009), 299-308.

[5] S. P. Goyal, P. Goswami and H. Silverman, Subordination and superordination results for a class of analytic multivalent functions, Int. J. Math. Math. Sci. 2008, Art. ID 561638, 12 pp.

[6] R. W. Ibrahim and M. Darus, On a univalent class involving differential subordination with applications, J. Math. Statistics 7(2) (2011), 137-143.

[7] N. Magesh, G. Murugusundaramoorthy, T. Rosy and K. Muthunagai, Subordination and superordination for analytic functions associated with convolution structure, Int. J. Open Problems Complex Analysis 2(2) (2010), 67-81.

[8] S. S. Miller and P. T. Mocanu, Differential subordinations: theory and applications, Series on Monographs and Textbooks in Pure and Applied Mathematics, Vol. 225, Marcel Dekker, Inc., New York and Basel, 2000.

[9] G. Murugusundaramoorthy and N. Magesh, Differential sandwich theorems for analytic functions defined by Hadamard product, Ann. Univ. Mariae. Curie-Sklodowska Sect. A 61 (2007), 117-127.

[10] G. Murugusundaramoorthy and N. Magesh, Differential subordinations and superordinations for analytic functions defined by convolution structure, Stud. Univ. Babeş-Bolyai Math. 54(2) (2009), 83-96.

[11] G. S. Sălăgean, Subclasses of univalent functions, Lecture Notes in Math., 1013, Springer Verlag, Berlin, 1983, pp. 362-372.

[12] H. Srivastava and S. S. Eker, Some applications of a subordination theorem for a class of analytic functions, Appl. Math. Letters 21(4) (2008), 394-399.

[13] S. R. Swamy, Inclusion properties of certain subclasses of analytic functions, Int. Math. Forum 7(36) (2012), 1751-1760. 
[14] A. K. Wanas, Differential sandwich theorems for integral operator of certain analytic functions, Gen. Math. Notes 15(1) (2013), 72-83.

[15] A. K. Wanas, On sandwich theorems for higher-order derivatives of multivalent analytic functions associated with the generalized Noor integral operator, Asian-Eur. J. Math. 8(1) (2015), 1450024, 14 pp.

[16] A. K. Wanas and A. S. Joudah, Sandwich theorems for certain subclasses of analytic functions defined by convolution structure with generalized operator, An. Univ. Oradea Fasc. Mat. 21(1) (2014), 183-190.

[17] A. K. Wanas and A. H. Majeed, Differential sandwich theorems for multivalent analytic functions defined by convolution structure with generalized hypergeometric function, $A n$. Univ. Oradea Fasc. Mat. 25(2) (2018), 37-52. 